Chinese Journal of Organic Chemistry

\title{
二茂铁修饰卟啉及含氟卟啉的光物理与电化学性质
}

\author{
苏 敏 李 晴 王亚光 陈树峰 赵海英* 边占喜 \\ (内蒙古大学化学化工学院 呼和浩特 010021)
}

\begin{abstract}
摘要 合成了二茂铁修饰的卟啉与含有三氟甲基的卟啉. 拉电子的三氟甲基使卟啉的紫外吸收发生蓝移，而给电子的 二茂铁基则使卟啉的紫外吸收发生红移. 在稳态荧光光谱中，二茂铁对卟啉苂光的猝灭程度大于三氟甲基，其中含有 双二茂铁基的卟啉发生了 $66 \%$ 的荧光猝灭, 量子产率 $\Phi_{\mathrm{f}}$ 仅为 0.08 , 说明在激发态卟啉和二茂铁之间发生了较强的电子 和能量的传递, 这些结果在时间分辨荧光光谱研究中进一步被证实. 电化学性质研究表明三氟甲基的引入使卟啉难失 去电子, 增加了卟啉的稳定性; 二茂铁的给电子作用使卟啉更容易被氧化，是很好的电子给体. 这些性质为此类化合 物进一步应用于给受体体系的构筑提供了一定的理论依据.
\end{abstract}

关键词 二茂铁; 含氟卟啉; 光谱性质; 电化学

\section{Photophysical and Electrochemical Studies of Porphyrin Containing Ferrocene and/or Fluorine}

\author{
Su, Min Li, Qing Wang, Yaguang Chen, Shufeng Zhao, Haiying* Bian, Zhanxi \\ (College of Chemistry and Chemical Engineering, Inner Mongolia University, Huhhot 010021)
}

\begin{abstract}
Functionalized porphyrin and porphyrin containing trifluoromethyl with ferrocene were synthesized. The UV-Vis absorption bands of porphyrin with an electron withdrawing trifluoromethyl group were blue-shifted and those of porphyrin with an electron donating ferrocenyl group were red-shifted. The porphyrin containing ferrocenyl exhibited strong fluorescence quenching compared with those containing trifluoromethyl. The quenching efficiency of diferrocenyl porphyrin was about $66 \%$ with a quantum yield $\Phi_{\mathrm{f}}$ of 0.08 . These results indicated that there was a strong interaction between the excited state of porphyrin and ferrocene, which was further confirmed by the time-resolved fluorescence measurements. The electrochemical studies indicated that it was difficult to lost electron for the stable porphyrin with an electron withdrawing trifluoromethyl. However, the porphyrin with a ferrocenyl group was oxidized easily to lost electron and was a good electron donor. The research is supposed to provide some theoretical basis for this kind of compound using in donor-acceptor system.
\end{abstract}

Keywords ferrocene; porphyrin containing fluorine; spectroscopic property; electrochemistry

卟啉类化合物具有特殊的二维共轭 $\pi$-电子结构、较 小的 HOMO-LUMO 能带以及很强的可见光吸收和发光 等性能, 是非常理想的给-受体(D-A)体系中的给电子部 分 ${ }^{[1]}$. 近年来, 利用卟啉的特殊电子结构和光电性能, 结合电子受体, 模拟植物体内光合反应过程, 以及设计 合成光电功能材料及器件, 从而实现对太阳能的有效合 理利用, 已成为国内外十分活跃的研究领域 ${ }^{[2 \sim 4]}$. 在这 些研究中, 关键的一步是通过给受体之间的光诱导电子 转移(PET) 以产生长寿命的电荷分离态 ${ }^{[5]}$. 但是, 由单一 的卟啉作为电子给体形成的给受体系统, 其电荷分离态 寿命较短, 所以大大影响了材料和器件的性能. 因此,
将卟啉连接上其它发色团如三苯胺、二茂铁、苯四甲酰 二亚胺以及另一金属卟啉单元等作为次级电子给体，通 过多步 PET 将电子传递给受体, 成为形成长寿命电荷分 离态的重要思路之一 ${ }^{[6 ~ 14]}$. 另一方面, 含氟有机分子由 于氟原子的较大电负性及化学惰性, 赋予有机化合物及 材料较高的稳定性与独特的性能, 用于液晶材料、光敏 材料以及生物与化学传感等, 有机氟化学近年来受到化 学界的极大关注 ${ }^{[15 ~ 19]}$. 本文用二茂铁修饰卟啉及含有 三氟甲基的卟啉(图 1)，通过紫外-可见吸收光谱 (UV-Vis)、稳态和时间分辨荧光光谱以及循环伏安法研 究二茂铁和三氟甲基的引入对卟啉光物理和电化学性

* E-mail: hyzhao@imu.edu.cn

Received January 24, 2013; revised March 14, 2013; published online March 22, 2013.

Project supported by the National Natural Science Foundation of China (Nos. 21102068, 21262023).

国家自然科学基金(Nos. 21102068, 21262023)资助项目。 
质的影响，为此类化合物进一步应用于 $\mathrm{D}-\mathrm{A}$ 体系的构筑 提供了一定的理论依据.

\section{1 结果与讨论}

\section{1 合成与表征}

用双氨基卟啉 $\mathbf{a}$ 分别与三氟乙酸酐和二茂铁甲酰氯 在室温下反应, 得到含氟卟啉 $\mathbf{c}$ 和双二茂铁取代的卟啉 $\mathbf{g}$; 用带有一个二茂铁基的氨基卟啉 $\mathbf{d}$ 和三氟乙酸䣶反 应，得到二茂铁修饰的含氟卟啉 f. 二茂铁和三氟甲基 的引入, 对卟啉的核磁共振光谱产生了一定的影响. 双 乙酰氨基卟啉 $\mathbf{b}$ 中酰胺质子的化学位移为 $\delta 7.51$, 而双 二茂铁甲酰基卟啉 $\mathbf{g}$ 中由于茂环的去屏蔽作用使得酰胺 质子的化学位移向低场移动, 出现在 $\delta 7.78$ 处; 化合物 c 中, 双三氟乙酰胺基质子的化学位移由于三氟甲基的 强拉电子作用移向更低场, 出现在 $\delta 8.24$ 处. 同样, 在 ${ }^{13} \mathrm{C} \mathrm{NMR}$ 中, 卟啉 $\mathbf{b}$ 的乙酰基甲基碳原子的化学位移为 $\delta 24.78$, 而卟啉 $\mathbf{c}$ 的三氟甲基碳原子的化学位移移至低 场, 出现在 $\delta 29.71$ 处.

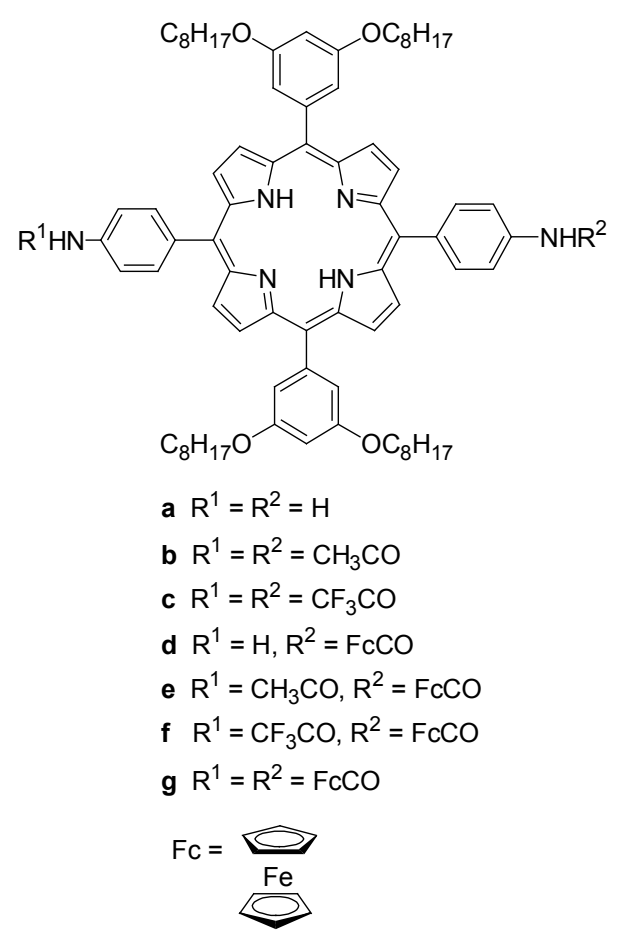

图 1 卟啉 $\mathbf{a} \sim \mathbf{g}$ 的结构

Figure 1 Structures of porphyrins $\mathbf{a} \sim \mathbf{g}$

\section{2 紫外-可见吸收光谱}

UV-Vis 光谱可反映出二茂铁或三氟甲基与卟啉在 基态电子相互作用的情况. 图 2 给出了卟啉 $\mathbf{g}, \mathbf{c}, \mathbf{f}$ 在 $N, N$-二甲基甲酰胺(DMF)中的 UV-Vis 光谱对照. 从图 2 中可以看出, 3 个化合物的吸收很接近, 但是最大吸收
处放大后可以看出一些细微的差别: 连有 2 个三氟甲基 的卟啉 $\mathbf{c}$ 紫外吸收发生了轻微的蓝移; 相反，二茂铁基 则使卟啉的吸收发生轻微的红移. 相对于卟啉 $\mathbf{c}$, 连有 1 个和 2 个二茂铁的卟啉 $\mathbf{f}$ 和 $\mathbf{g}$ 分别红移了 1 和 $2 \mathrm{~nm}$, 而 且吸收峰略有变宽. 这种小的扰动说明，二茂铁或三氟 甲基与卟啉在基态存在弱的相互作用. 三氟甲基的强拉 电子作用使卟啉 $\pi$ 电子的 HOMO 和 LUMO 能级差增大, 在紫外吸收表现为蓝移; 而二茂铁本身是较好的电子给 体，连接二茂铁后，扩大了卟啉的共轭体系，因此， $\pi$ 电 子的 HOMO 和 LUMO 能级差减小, 在紫外吸收表现为 红移 ${ }^{[20]}$.

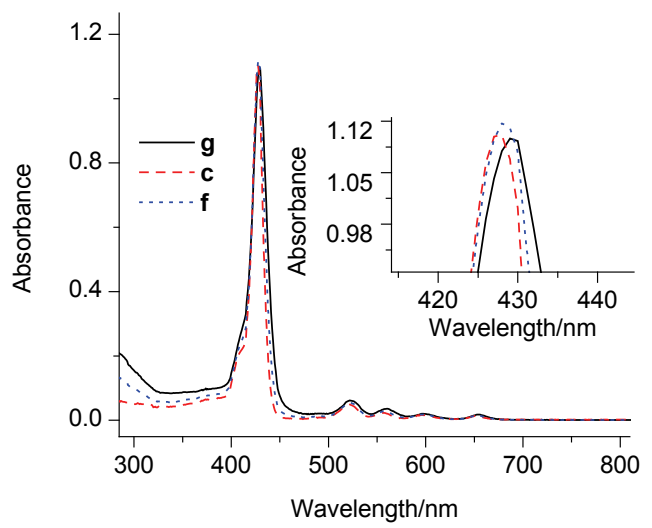

图 2 卟啉 $\mathbf{c}, \mathbf{f}, \mathbf{g}$ 在 DMF 中的 UV-Vis 光谱(插图是最大吸收 处的放大)

Figure 2 UV-Vis absorption spectra of $\mathbf{c}, \mathbf{f}, \mathbf{g}$ in DMF (insert shows the magnification of the maximum absorption)

\section{3 稳态及时间分辨荧光光谱}

为了研究激发态二茂铁和三氟甲基与卟啉之间的 电子相互作用情况，分别测试了卟啉 $\mathbf{c} \sim \mathbf{g}$ 的稳态与时 间分辨菼光光谱. 图 3 是卟啉 $\mathbf{c} \sim \mathbf{g}$ 在激发波长 $427 \mathrm{~nm}$ 处具有相同吸收时的稳态荧光光谱对照. 从图 3 可知, 除了卟啉 $\mathbf{c}$ 外, 其他几个化合物的较强苂光位于 653 和 $719 \mathrm{~nm}$ 处, 而 $\mathbf{c}$ 的苂光发生了 $2 \sim 3 \mathrm{~nm}$ 的蓝移, 出现在 650 和 $717 \mathrm{~nm}$ 处. 此外, 与卟啉 $\mathbf{b}$ 相比, 带有二茂铁和 三氟甲基的卟啉均发生了不同程度的荧光猝灭，为了定 量计算卟啉的的荧光猝灭程度，以 5,10,15,20-四苯基卟 啉(TPP, $\Phi=0.11, \mathrm{CHCl}_{3}$ 溶液)为标准, 通过比较的方法 计算了卟啉 $\mathbf{c} \sim \mathbf{g}$ 在 $\mathrm{DMF}$ 溶液中的荧光量子产率 ${ }^{[21]}$, 相 应化合物的菼光量子产率 $\left(\Phi_{\mathrm{f}}\right)$ 与猝灭率 $(Q)^{[22]}$ 见表 1 . 从 表 1 可以看出, 卟啉 $\mathbf{b}$ 的 $\Phi_{\mathrm{f}}$ 为 0.23 , 两个三氟甲基 $(\mathbf{c}$, $\left.\Phi_{\mathrm{f}}=0.17, Q=27 \%\right)$ 与一个二茂铁基 $\left(\mathbf{e}, \Phi_{\mathrm{f}}=0.16, Q=\right.$ $31 \%$ )对卟啉的荧光猝灭程度接近，而带有两个二茂铁 $\mathbf{g}$ 的卟啉荧光发生了 $66 \%$ 的猝灭, $\Phi_{\mathrm{f}}$ 仅为 0.08 . 这些结果 说明二茂铁和三氟甲基均与卟啉在激发态存在较强分 子内电子或能量转移相互作用, 而二茂铁与卟啉之间的 
作用更大.

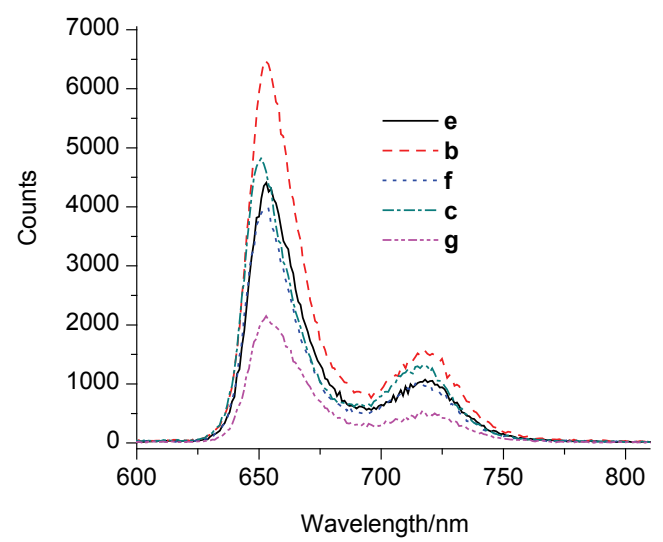

图 3 吓啉 $\mathbf{c} \sim \mathbf{g}$ 的 DMF 溶液在激发波长 $427 \mathrm{~nm}$ 处具有相同 吸收的苂光光谱

Figure 3 Fluorescence spectra of $\mathbf{c} \sim \mathbf{g}$ in DMF with normalization of the absorbance of the excitation wavelength $427 \mathrm{~nm}$ to the same value

表 1 卟啉 $\mathbf{b} \sim \mathbf{g}$ 的荧光猝灭率 $(Q)$ 、荧光量子产率 $\left(\Phi_{\mathrm{f}}\right)$ 及苂光 寿命 $\left(\tau_{\mathrm{f}}\right)$

Table 1 Quenching ratio $(Q)$, fluorescence quantum yields $\left(\Phi_{\mathrm{f}}\right)$ and fluorescence lifetime $\left(\tau_{\mathrm{f}}\right)$ of compounds $\mathbf{b} \sim \mathbf{g}$

\begin{tabular}{cccc}
\hline Compound & $Q / \%$ & $\Phi_{\mathrm{f}}$ & $\tau_{\mathrm{f}} / \mathrm{ns}$ (fraction) \\
\hline $\mathbf{b}$ & - & 0.23 & $12.47(100 \%)$ \\
$\mathbf{c}$ & 27 & 0.17 & $12.77(100 \%)$ \\
$\mathbf{e}$ & 31 & 0.16 & $12.04(100 \%)$ \\
$\mathbf{f}$ & 39 & 0.14 & $12.24(100 \%)$ \\
$\mathbf{g}$ & 66 & 0.08 & $11.44(100 \%)$ \\
\hline
\end{tabular}

二茂铁和三氟甲基与卟啉之间的相互作用在时间 分辨荧光光谱中也得到了确证. 图 4 给出了卟啉 $\mathbf{g}, \mathbf{c}, \mathbf{e}$ 的时间分辨苂光衰减曲线, 他们都表现为单指数衰减, 用单指数方程进行拟合得出卟啉激发单线态荧光寿命,

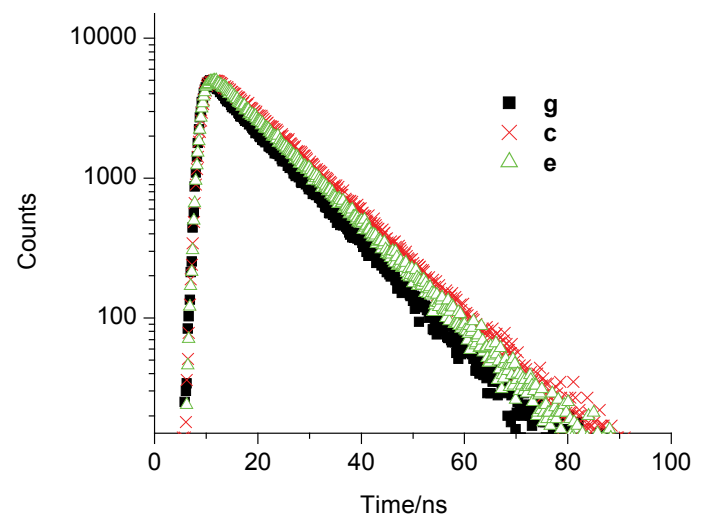

图 4 卟啉 $\mathbf{c}, \mathbf{e}$ 和 $\mathbf{g}$ 在 $\mathrm{DMF}$ 中的苂光衰减曲线 $\left(\lambda_{\mathrm{ex}}=427 \mathrm{~nm}\right.$, $\lambda_{\mathrm{em}}=653 \mathrm{~nm}$ )

Figure 4 Time profiles of fluorescence lifetime measurement of c, e and $\mathbf{g}$ in DMF $\left(\lambda_{\mathrm{ex}}=427 \mathrm{~nm}, \lambda_{\mathrm{em}}=653 \mathrm{~nm}\right)$
具体数据列于表 1 . 从表 1 可以看出, 连有 2 个三氟甲基 $\mathbf{c}$ 的荧光寿命比 $\mathbf{b}$ 的略长, $\mathbf{f}$ 比 $\mathbf{e}$ 的荧光寿命也略长, 说 明拉电子的三氟甲基使卟啉的苂光衰减速度减慢; 而给 电子的二茂铁基使卟啉的荧光衰减速度加快, 带有 2 个 二茂铁基卟啉 $\mathbf{g}$ 的荧光寿命最短, 仅为 $11.44 \mathrm{~ns}$, 说明二 茂铁和卟啉之间发生较强的电子或能量的传递 ${ }^{[23]}$.

\section{4 电化学性质}

为了研究二茂铁与三氟甲基对卟啉电化学性质的 影响, 用循环伏安法测试了卟啉 $\mathbf{b} \sim \mathbf{g}$ 的氧化还原电势, 结果列于表 2. 从表 2 数据可知, 卟啉 $\mathbf{b}$ 和含氟卟啉 $\mathbf{c}$ 都出现了四对氧化还原电位, 而连接上二茂铁的卟啉 $\mathbf{e} \sim \mathbf{g}$, 除了卟啉的四对氧化还原电势外, 又在比卟啉第 一氧化电势低的位置 $0.17 \sim 0.22 \mathrm{~V}$ 附近出现了 1 个归属 于二茂铁的氧化还原电势, 说明二茂铁作为电子给体能 够将电子转移给卟啉. 此外, 通过对卟啉的第一氧化电 势数据进行对比, 发现连有 2 个三氟甲基的 $\mathbf{c}$ 比未连三 氟甲基 $\mathbf{b}$ 的第一氧化电势高 $55 \mathrm{mV}$, 而连有 1 个三氟甲 基 $\mathbf{f}$ 的第一氧化电势比 $\mathbf{e}$ 也高 $14 \mathrm{mV}$, 说明三氟甲基的 引入使吓啉难失去电子，增加了吓啉的稳定性; 相反, 连有二茂铁的 $\mathbf{e}$ 和 $\mathbf{f}$ 中吓啉的第一氧化电势都比 $\mathbf{b}$ 的低, 说明二茂铁的给电子作用使吓啉更容易失去电子而被 氧化，因此这类卟啉在 D-A 体系中可以作为优良的电子 给体和光敏材料.

表 2 卟啉 $\mathbf{b} \sim \mathbf{g}$ 的在 $\mathrm{CH}_{2} \mathrm{Cl}_{2}$ 溶液中的氧化还原电势 Table 2 Electrochemical redox potentials of $\mathbf{b} \sim \mathbf{g}$ in $\mathrm{CH}_{2} \mathrm{Cl}_{2}$

\begin{tabular}{cccccc}
\hline \multirow{2}{*}{ Compd. } & \multicolumn{5}{c}{ Potential/V vs. Fc/Fc } \\
\cline { 2 - 6 } & $\mathrm{P}^{-/ 2-}$ & $\mathrm{P}^{0 /-}$ & $\mathrm{Fc}^{0 /+}$ & $\mathrm{P}^{0 /+}$ & $\mathrm{P}^{+/ 2+}$ \\
\hline b & -2.108 & -1.749 & - & 0.494 & 0.777 \\
$\mathbf{c}$ & -2.110 & -1.754 & - & 0.549 & 0.847 \\
$\mathbf{e}$ & -2.122 & -1.780 & 0.224 & 0.485 & 0.764 \\
$\mathbf{f}$ & -2.124 & -1.782 & 0.174 & 0.499 & 0.811 \\
$\mathbf{g}$ & -2.129 & -1.774 & 0.187 & 0.483 & 0.780 \\
\hline
\end{tabular}

\section{2 结论}

合成了二茂铁和三氟甲基单独或共同修饰的卟啉. 研究发现给电子的二茂铁和拉电子的三氟甲基对卟啉 的光物理和电化学性质产生相反的作用. 拉电子的三氟 甲基使卟啉的紫外吸收发生轻微蓝移，而给电子的二茂 铁基则使吓啉的吸收发生轻微红移，说明在基态二茂 铁、三氟甲基与卟啉之间存在弱的相互作用. 在稳态荧 光光谱中, 二茂铁对卟啉荧光的猝灭程度大于三氟甲 基，其中含有双二茂铁基的卟啉发生了 $66 \%$ 的荧光猝 灭, 量子产率 $\Phi_{\mathrm{f}}$ 仅为 0.08 ; 时间分辨荧光光谱研究发现 三氟甲基使卟啉的苂光衰减速度略微减慢; 而二茂铁基 使卟啉的苂光衰减速度加快, 荧光寿命缩短. 这些结果 说明在激发态卟啉和二茂铁之间发生了较强的电子或 
能量的传递. 电化学性质研究表明三氟甲基的引入使卟 啉难失去电子, 增加了卟啉的稳定性; 二茂铁的给电子 作用使卟啉更容易被氧化, 是很好的电子给体. 这些研 究此类化合物进一步应用于 D-A 体系的构筑提供了一 定的理论依据.

\section{3 实验部分}

\section{1 仪器与试剂}

NEXUS-670 傅里叶变换红外光谱仪, $\mathrm{KBr}$ 压片; Avance Bruker (500 MHz)核磁共振仪, 以 TMS 为内标; Analytikjena SPECORD.50 UV-Vis 光谱仪; IonSpec 710T FTICR/MS 高分辨质谱仪(MALDI 源). Edinburgh Instruments FLS920 稳态和时间分辨苂光光谱仪, 用时间 相关单光子计数(TCSPC)方法, NF900 氢灯作为光源, 测试前样品需置于带有塞子的 $1 \mathrm{~cm}$ 石英池中并通氮气 饱和 $15 \mathrm{~min}$; MEC-12B 电化学分析仪, 在 $298 \mathrm{~K}$ 下, 样 品溶于干燥的 $\mathrm{CH}_{2} \mathrm{Cl}_{2}$ 溶液, 加入 $0.1 \mathrm{~mol} / \mathrm{L}$ 四丁基六氟 磷酸氨 $\left(\mathrm{TBAPF}_{6}\right)$ 作为支持电解质, 用三电极方法, 玻碳 电极为工作电极, $\mathrm{Pt}$ 为对电极, $\mathrm{Ag} / \mathrm{Ag}^{+}$为参比电极, $\mathrm{Fc} / \mathrm{Fc}^{+}$作为外标. 所用试剂均为市售分析纯试剂, 溶剂 的干燥按常规方法进行.

\section{2 实验方法}

本文所研究化合物的结构如图 1 所示, 其中化合物 a, b 按文献[9]方法合成, 化合物 d, e 按文献[10]方法合 成.

5,15-双(4-三氟乙酰胺基苯基)-10,20-双(3,5-二辛氧 基苯基)卟啉(c)的合成：卟啉 $\mathbf{a}(15 \mathrm{mg}, 0.013 \mathrm{mmol}$ )溶于 $6 \mathrm{~mL}$ 甲苯和 $3 \mathrm{~mL}$ 吡啶, 加入三氟乙酸酐 $(20 \mathrm{mg}, 0.26$ $\mathrm{mmol}$ )和 4-二甲基氨基吡啶(DMAP) (3 mg, $0.025 \mathrm{mmol}$ ), $\mathrm{N}_{2}$ 保护下室温搅拌 $2 \mathrm{~h}$. 减压蒸除溶剂, 过硅胶柱, $\mathrm{CH}_{2} \mathrm{Cl}_{2}$ 洗脱, 得到 $11 \mathrm{mg}$ 紫色粉末 $\mathbf{c}$, 产率 $63 \%$. UV-Vis $\left(\mathrm{CHCl}_{3}\right) \lambda_{\max }(\lg \varepsilon): 428$ (5.61), 522 (4.23), 557 (3.83), 597 (3.79), 651 (3.58) nm; Fluorescence $\left(\mathrm{CHCl}_{3}, \lambda_{\mathrm{ex}}=425 \mathrm{~nm}\right)$ $\lambda_{\mathrm{em}}: 650,714 \mathrm{~nm} ;{ }^{1} \mathrm{H} \mathrm{NMR}\left(\mathrm{CDCl}_{3}, 400 \mathrm{MHz}\right) \delta: 9.00(\mathrm{~d}$, $J=4.69 \mathrm{~Hz}, 4 \mathrm{H}, \beta$-pyrrole-H), 8.80 (d, $J=4.69 \mathrm{~Hz}, 4 \mathrm{H}$, $\beta$-pyrrole-H), 8.26 (d, $J=8.42 \mathrm{~Hz}, 4 \mathrm{H}$, phenyl-H), 8.24 (s, 2H, CONH), 8.01 (d, $J=8.42 \mathrm{~Hz}, 4 \mathrm{H}$, phenyl-H), 7.38 (s, $4 \mathrm{H}$, phenyl-H), 6.90 (s, 2H, phenyl-H), 4.13 (t, $J=6.57$ $\left.\mathrm{Hz}, 8 \mathrm{H}, \mathrm{OCH}_{2}\right), 1.88 \sim 1.83\left(\mathrm{~m}, 8 \mathrm{H}, \mathrm{CH}_{2}\right), 1.52 \sim 1.46$ $\left(\mathrm{m}, 8 \mathrm{H}, \mathrm{CH}_{2}\right), 1.40 \sim 1.26\left(\mathrm{~m}, 32 \mathrm{H}, \mathrm{CH}_{2}\right), 0.87$ (t, $J=6.78$ $\left.\mathrm{Hz}, 12 \mathrm{H}, \mathrm{CH}_{3}\right),-2.83$ (s, 2H, pyrrole-NH); ${ }^{13} \mathrm{C} \mathrm{NMR}$ $\left(\mathrm{CDCl}_{3}, 500 \mathrm{MHz}\right) \delta: 158.35,143.66,140.32,135.24$, $134.89,120.39$, 118.68, 118.61, 117.00, 114.71, 114.43, $101.03,68.39,31.80,29.71,29.39$, 29.37, 29.24, 26.11, 22.65, 14.09; IR (KBr) v: 3318, 2925, 2855, 1734, 1594,
1161, $800 \mathrm{~cm}^{-1}$; HRMS calcd for $\mathrm{C}_{80} \mathrm{H}_{95} \mathrm{~F}_{6} \mathrm{~N}_{6} \mathrm{O}_{6}[\mathrm{M}+\mathrm{H}]^{+}$ 1349.7217, found 1349.7215 .

5-(4-二茂铁甲酰胺基苯基)-15-(4-三氟乙酰胺基苯 基)-10,20-双(3,5-二辛氧基苯基)卟啉(f)的合成：卟啉 $\mathbf{d}$ $(11 \mathrm{mg}, 0.008 \mathrm{mmol})$ 溶于 $6 \mathrm{~mL}$ 甲苯和 $3 \mathrm{~mL}$ 吡定, 加入 乙酰氯 $(6 \mathrm{mg}, 0.08 \mathrm{mmol})$ 和 DMAP (1.8 mg, $0.015 \mathrm{mmol})$, $\mathrm{N}_{2}$ 保护下室温搅拌 $2 \mathrm{~h}$. 减压蒸除溶剂, 过硅胶柱, $\mathrm{CH}_{2} \mathrm{Cl}_{2}$ 洗脱, 得到 $8 \mathrm{mg}$ 紫色粉末 $\mathbf{f}$, 产率 $68 \%$. UV-Vis $\left(\mathrm{CHCl}_{3}\right) \lambda_{\max }(\lg \varepsilon): 428$ (5.91), 521 (4.61), 558 (4.31), 597 (4.12), 653 (4.04) nm; Fluorescence $\left(\mathrm{CHCl}_{3}, \lambda_{\mathrm{ex}}=425 \mathrm{~nm}\right)$ $\lambda_{\mathrm{em}}: 651,714 \mathrm{~nm} ;{ }^{1} \mathrm{H} \mathrm{NMR}\left(\mathrm{CDCl}_{3}, 500 \mathrm{MHz}\right) \delta: 8.98(\mathrm{~d}$, $J=4.30 \mathrm{~Hz}, 4 \mathrm{H}, \beta$-pyrrole-H), 8.92 (d, $J=4.45 \mathrm{~Hz}, 2 \mathrm{H}$, $\beta$-pyrrole-H), 8.80 (d, $J=4.30 \mathrm{~Hz}, 2 \mathrm{H}, \beta$-pyrrole-H), 8.25 (d, $J=8.28 \mathrm{~Hz}, 2 \mathrm{H}$, phenyl-H), 8.23 (s, 1H, CONH), 8.20 (d, $J=8.28 \mathrm{~Hz}, 2 \mathrm{H}$, phenyl-H), 8.01 (t, $J=8.34 \mathrm{~Hz}, 4 \mathrm{H}$, phenyl-H), 7.74 (s, 1H, CONH), 7.37 (s, 4H, phenyl-H), 6.89 (s, 2H, phenyl-H), 4.94 (s, 2H, FcH), 4.53 (s, 2H, $\mathrm{FcH}), 4.40$ (s, 5H, FcH), $4.12\left(\mathrm{t}, J=6.50 \mathrm{~Hz}, 8 \mathrm{H}, \mathrm{CH}_{2} \mathrm{O}\right)$, $1.88 \sim 1.83\left(\mathrm{~m}, 8 \mathrm{H}, \mathrm{CH}_{2}\right), 1.52 \sim 1.46\left(\mathrm{~m}, 8 \mathrm{H}, \mathrm{CH}_{2}\right)$, $1.38 \sim 1.27\left(\mathrm{~m}, 32 \mathrm{H}, \mathrm{CH}_{2}\right), 0.85(\mathrm{t}, J=6.50 \mathrm{~Hz}, 12 \mathrm{H}$, $\left.\mathrm{CH}_{3}\right),-2.81$ (s, $2 \mathrm{H}$, pyrrole-NH); ${ }^{13} \mathrm{C} \mathrm{NMR}\left(\mathrm{CDCl}_{3}, 500\right.$ MHz) $\delta: 169.01,158.31,143.77,140.41,137.87,135.24$, $134.83,120.23,119.76,118.66,118.29,117.96,114.39$, 101.00, 71.13, 70.04, 68.40, 68.37, 31.80, 29.71, 29.39, 29.37, 29.24, 26.11, 22.65, 14.10; IR (KBr) v: 3319, 3095, 2923, 2853, 1738, 1650, 1592, 1261, 1161, 1023, 801 $\mathrm{cm}^{-1}$; HRMS calcd for $\mathrm{C}_{89} \mathrm{H}_{104} \mathrm{~F}_{3} \mathrm{FeN}_{6} \mathrm{O}_{6}[\mathrm{M}+\mathrm{H}]$ 1465.7319, found 1465.7315 .

5,15-双(4-二茂铁甲酰胺基苯基)-10,20-双(3,5-二辛 氧基苯基)卟啉 $(\mathbf{g})$ 的合成：双氨基卟啉 $\mathbf{a}(8 \mathrm{mg}, 0.007$ $\mathrm{mmol})$ 溶于 $5 \mathrm{~mL}$ 甲苯和 $3.5 \mathrm{~mL}$ 吡啶, 加入 DMAP (1.65 $\mathrm{mg}, 0.014 \mathrm{mmol})$, 室温下滴加溶有二茂铁甲酰氯(6.9 $\mathrm{mg}, 0.028 \mathrm{mmol}$ )的甲苯溶液, $\mathrm{N}_{2}$ 保护下室温搅拌 $1 \mathrm{~h}$. 减 压蒸除溶剂, 过硅胶柱, $\mathrm{CH}_{2} \mathrm{Cl}_{2}$ 洗脱, 得到 $5.8 \mathrm{mg}$ 紫色 粉末 $\mathbf{g}$, 产率 53\%. UV-Vis $\left(\mathrm{CHCl}_{3}\right) \lambda_{\max }(\mathrm{lg} \varepsilon): 429$ (5.44), 523 (4.11), 560 (3.84), 597 (3.67), 653 (3.54) nm; Fluorescence $\left(\mathrm{CHCl}_{3}, \lambda_{\mathrm{ex}}=425 \mathrm{~nm}\right) \lambda_{\mathrm{em}}: 653,716 \mathrm{~nm} ;{ }^{1} \mathrm{H}$ NMR $\left(\mathrm{CDCl}_{3}, 400 \mathrm{MHz}\right) \delta$ : $9.00(\mathrm{~d}, J=4.63 \mathrm{~Hz}, 4 \mathrm{H}, \beta$-pyrroleH), $8.92(\mathrm{~d}, J=4.63 \mathrm{~Hz}, 4 \mathrm{H}, \beta$-pyrrole-H), 8.22 (d, $J=$ $8.28 \mathrm{~Hz}, 4 \mathrm{H}$, phenyl-H), 8.02 (d, $J=8.28 \mathrm{~Hz}, 4 \mathrm{H}$, phenyl$\mathrm{H}), 7.78$ (s, 2H, CONH), 7.40 (s, 4H, phenyl-H), 6.91 (s, 2H, phenyl-H), 4.96 (s, 4H, FcH), 4.54 (s, 4H, FcH), 4.42 $(\mathrm{s}, 10 \mathrm{H}, \mathrm{FcH}), 4.14\left(\mathrm{t}, J=6.49 \mathrm{~Hz}, 8 \mathrm{H}, \mathrm{CH}_{2} \mathrm{O}\right), 1.88 \sim 1.83$ $\left(\mathrm{m}, 8 \mathrm{H}, \mathrm{CH}_{2}\right), 1.52 \sim 1.46\left(\mathrm{~m}, 8 \mathrm{H}, \mathrm{CH}_{2}\right), 1.38 \sim 1.27(\mathrm{~m}$, $\left.32 \mathrm{H}, \mathrm{CH}_{2}\right), 0.87\left(\mathrm{t}, J=6.56 \mathrm{~Hz}, 12 \mathrm{H}, \mathrm{CH}_{3}\right),-2.77(\mathrm{~s}, 2 \mathrm{H}$, 
pyrrole-NH); ${ }^{13} \mathrm{C} \mathrm{NMR}\left(\mathrm{CDCl}_{3}, 500 \mathrm{MHz}\right) \delta: 168.98$, $158.31,143.9,137.85,135.21,120.08,119.45,117.96$, $114.39,101.05,71.11,70.03,68.41,68.37,31.80,29.39$, 29.23, 26.11, 22.64, 14.10; IR (KBr) v: 3317, 3095, 2923, 2854, 1650, 1589, 1509, 1261, 1161, 1056, $800 \mathrm{~cm}^{-1}$; HRMS calcd for $\mathrm{C}_{98} \mathrm{H}_{113} \mathrm{Fe}_{2} \mathrm{~N}_{6} \mathrm{O}_{6}[\mathrm{M}+\mathrm{H}]^{+} 1582.7454$, found 1582.7660 .

\section{References}

[1] Guldi, D. M.; Rahman, G. M. A.; Sgobba, V.; Ehli, C. Chem. Soc. Rev. 2006, 35, 471.

[2] Fungo, F.; Milanesio, M. E.; Durantini, E. N.; Otero, L.; Dittrich, T. J. Mater. Chem. 2007, 17, 2107.

[3] Straight, S. D.; Andréasson, J.; Kodis, G.; Moore, A. L.; Moore, T. A.; Gust, D. J. Am. Chem. Soc. 2005, 127, 2717.

[4] Fukuzumi, S.; Kojima, T. J. Mater. Chem. 2008, 18, 1427.

[5] Imahori, H.; Sekiguchi, Y.; Kashiwagi, Y.; Sato, T.; Araki, Y.; Ito, O.; Yamada, H.; Fukuzumi, S. Chem. Eur. J. 2004, 10, 3184.

[6] D'Souza, F.; Gadde, S.; Islam, D. M. S.; Wijesinghe, C. A.; Schumacher, A. L.; Zandler, M. E.; Araki, Y.; Ito, O. J. Phys. Chem. A 2007, 111, 8552 .

[7] Imahori, H.; Tamaki, K.; Yamada, H.; Yamada, K.; Sakata, Y.; Nishimura, Y.; Yamazaki, I.; Fujitsuka, M.; Ito, O. Carbon 2000, 38, 1599.

[8] Imahori, H.; Tamaki, K.; Guldi, D. M.; Luo, C.; Fujitsuka, M.; Ito, O.; Sakata, Y.; Fukuzumi, S. J. Am. Chem. Soc. 2001, 123, 2607.

[9] Zhao, H. Y.; Chen, C.; Zhu, Y. Z.; Shi, M. Z.; Zheng, J. Y. J.
Nanopart. Res. 2012, DOI: 10.1007/s11051-012-0765-0.

[10] Zhao, H. Y.; Zhu, Y. Z.; Chen, C.; He, L.; Zheng, J. Y. Carbon 2012, 50, 4894.

[11] Galloni, P.; Floris, B.; Cola, L. D.; Cecchetto, E.; Williams, R. M. J. Phys. Chem. C 2007, 111, 1517.

[12] Samanta, S.; Sengupta, K.; Mittra, K.; Bandyopadhyay, S.; Dey, A. Chem. Commun. 2012, 48, 7631.

[13] Zhu, P.; Ma, P.; Wang, Y.; Wang, Q.; Zhao, X.; Zhang, X. Eur. J. Inorg. Chem. 2011, 4241.

[14] Rohde, G. T.; Sabin, J. R.; Barrett, C. D.; Nemykin, V. N. New J. Chem. 2011, 35, 1440.

[15] Zhou, X. L.; Kang, S. W.; Kumar, S.; Li, Q. Liq. Cryst. 2009, 36, 269.

[16] Sakurai, T.; Tashiro, K.; Honsho, Y.; Saeki, Akinori.; Seki, Shu.; Osuka, A.; Muranaka, A.; Uchiyama, M.; Kim, J.; Ha, S.; Kato, K.; Takata, M.; Aida, T. J. Am. Chem. Soc. 2011, 133, 6537.

[17] Kim, E. H.; Kadkin, O. N.; Kim, S. Y.; Choi, M.-G. Eur. J. Inorg. Chem. 2011, 2933.

[18] Kim, S. Y.; Kadkin, O. N.; Kim, E. H.; Choi, M.-G. J. Organomet. Chem. 2011, 696, 2429.

[19] Bonar-Law, R. P. J. Org. Chem. 1996, 61, 3623.

[20] He, D.; Zhang, H.; Peng, Y.; Ma, D.; Wang, Y.; Yang, H.; Chen, W.; Zhang, T. Chin. J. Org. Chem. 2012, 32, 1320 (in Chinese). (贺丹丹, 张宏, 彭亦如, 马冬冬, 王瑜华, 杨洪钦, 陈婉玲, 张 甜甜, 有机化学, 2012, 32, 1320.)

[21] Ormond, A. B.; Freeman, H. S. Dyes Pigm. 2013, 96, 440.

[22] Xu, H.; Zhu, Y. Z.; Zheng, J. Y. Supramol. Chem. 2007, 19, 365.

[23] D'Souza, F.; Smith, P. M.; Gadde, S.; McCarty, A. L.; Kullman, M. J.; Zandler, M. E.; Itou, M.; Araki, Y.; Ito, O. J. Phys. Chem. B 2004, 108, 11333.

(Qin, X.) 